ARTICLE

\title{
Temperature Dependence of Li-Glass Scintillator Response to Neutrons
}

\author{
Yuki OSHIMA $^{1, *}$, Takashi YASUMUNE ${ }^{1}$, Takurou MASUDA $^{1}$, Keisuke MAEHATA $^{1}$, \\ Kenji ISHIBASHI ${ }^{1}$ and Takahiro $\mathrm{UMENO}^{1,2}$ \\ ${ }^{1}$ Department of Applied Quantum Physics and Nuclear Engineering, Kyushu University \\ 744 Motooka, Nishi-ku, Fukuoka 819-0395, Japan \\ 2 Taiyo Nippon Sanso Corporation, Tsukuba, Japan
}

\begin{abstract}
A Li-glass scintillator was placed in the slow neutron field at temperatures of $300 \mathrm{~K}$ and $77 \mathrm{~K}$. An avalanche photodiode (APD) was employed for converting scintillation photons into voltage signal pulses. A peak corresponding to the Q-value of the ${ }^{6} \mathrm{Li}(n, \alpha) \mathrm{T}$ reaction appeared in both pulse height distributions obtained at temperatures of $300 \mathrm{~K}$ and $77 \mathrm{~K}$. By using the relationship between the Compton-edge energy and the pulse height in response of the Li-glass scintillator to $\gamma$-rays radiated from ${ }^{137} \mathrm{Cs}$, the values of the gamma equivalent energy of the thermal neutron peak were evaluated to be $1.60 \pm 0.09 \mathrm{MeV}$ and $1.16 \pm 0.06 \mathrm{MeV}$ at temperatures of $300 \mathrm{~K}$ and $77 \mathrm{~K}$, respectively. For the Li-glass scintillator, the ratio of the light yield in thermal neutron detection to that in $\gamma$-ray detection was found to decrease with temperatures.
\end{abstract}

KEYWORDS: radiation detection, Li-glass scintillator, avalanche photodiode, neutron, low temperature

\section{Introduction}

The europium activated crystal of ${ }^{6} \mathrm{LiI}$ is a solid scintillator which detects a slow neutron using the ${ }^{6} \mathrm{Li}(n, \alpha) \mathrm{T}$ reaction with the Q-value of $4.78 \mathrm{MeV}$. Although $\gamma$-rays exist with neutrons in many measurements, the high Q-value in the ${ }^{6} \mathrm{Li}(n, \alpha) \mathrm{T}$ reaction allows to discriminate between neutrons and $\gamma$-rays in detection signals of ${ }^{6} \mathrm{LiI}(\mathrm{Eu})$ scintillator. The energy resolution of ${ }^{6} \mathrm{LiI}(\mathrm{Eu})$ scintillator was found to be improved by lowering the temperature down to $77 \mathrm{~K} .{ }^{1)}$ However, handling of ${ }^{6} \mathrm{LiI}(\mathrm{Eu})$ crystal is difficult due to very strong deliquescence.

$\mathrm{A}^{6} \mathrm{Li}$-glass scintillator is employed to detect slow neutrons in various systems since the Li-glass is robust being resistant to all organic and inorganic chemicals except hydrofluoric acid. However, the light yield of Li-glass scintillator is smaller than that of $\mathrm{LiI}(\mathrm{Eu})$ scintillator. If light yield of Liglass scintillator grows with lowering temperatures, performance of Li-glass scintillator is expected to be improved in neutron detection.

In this work, the pulse height distributions of detection signals of Li-glass scintillator were obtained by irradiation with neutrons and $\gamma$-rays at temperatures of $300 \mathrm{~K}$ and $77 \mathrm{~K}$.

\section{Li-glass scintillator}

The Li-glass scintillator GS20 (APPLIED SCINTILLATION TECHNOLOGIES) is used in this work. The Li-glass scintillator is made of a silica glass with lithium oxide doped cerium as the activator. The isotopic ratio of ${ }^{6} \mathrm{Li}$ in the $\mathrm{Li}$-glass scintillator is enriched to be $95 \mathrm{~mol} \%$ for increasing sensitivity to thermal neutrons. Specifications of the Li-glass scintillator are summarized in Table $\mathbf{1}$.

Moderated neutrons induce the ${ }^{6} \operatorname{Li}(n, \alpha) \mathrm{T}$ reaction with ${ }^{6} \mathrm{Li}$ in the Li-glass scintillator. Scintillation light is emitted by excitation of Li-glass by the kinetic energy of alpha and

\footnotetext{
*Corresponding author, E-mail: no-0040@kune2a.nucl.kyushu-u.ac.jp

(C)Atomic Energy Society of Japan
}

Table 1 Specification of Li-glass scintillator (GS20).

\begin{tabular}{cc}
\hline Size & $10 \times 10 \times 5 \mathrm{~mm}$ \\
Isotope ratio of ${ }^{6} \mathrm{Li}$ & $95 \mathrm{~mol} \%$ \\
Wavelength of maximum emission & $395 \mathrm{~nm}$ \\
Decay time & $50-70 \mathrm{~ns}$ \\
Resolution on the thermal neutron peak & $15-28 \%$ \\
Light output relative to anthracene & $20-30 \%$ \\
Light output relative to NaI(Tl) & $9-13 \%$ \\
scintillator & \\
\hline
\end{tabular}

triton generated in the ${ }^{6} \mathrm{Li}(n, \alpha) \mathrm{T}$ reaction.

Since the atomic number of the elements in the glass is small, sensitivity of the Li-glass scintillator to the $\gamma$-rays is low. Continuous spectrum following Compton-edge appears in pulse height distribution of detection signals of Li-glass scintillator with irradiating $\gamma$-rays.

\section{Avalanche photodiode}

In this work, an avalanche photodiode (APD) was employed to convert scintillation light into electronic charge signal. The APD is a silicon photodiode with the multiplication mechanism. A large signal output can be obtained by charge amplification in the silicon semiconductor making a strong electric field. The APD exhibits strong temperature dependence of the operational performance. The performance of APD was found to be improved by lowering temperature down to $77 \mathrm{~K}^{2) 3}$ )

Figure 1 shows a schematic structure of APD (S86648099(X) Hamamatsu Photonics K.K.) used in this work. The depletion layer is formed in the APD by applying a reverse bias voltage. The steep potential gradient is generated in the avalanche area, and most of the reverse bias voltage to APD is impressed to avalanche area. Scintillation light enters through thin $\mathrm{p}^{+}$layer into $\pi\left(\mathrm{p}^{-}\right)$layer, electron-hole pairs are generated in $\pi\left(\mathrm{p}^{-}\right)$layer by deposited energy of scintillation light. Electrons are carried to the avalanche area by the electric field 


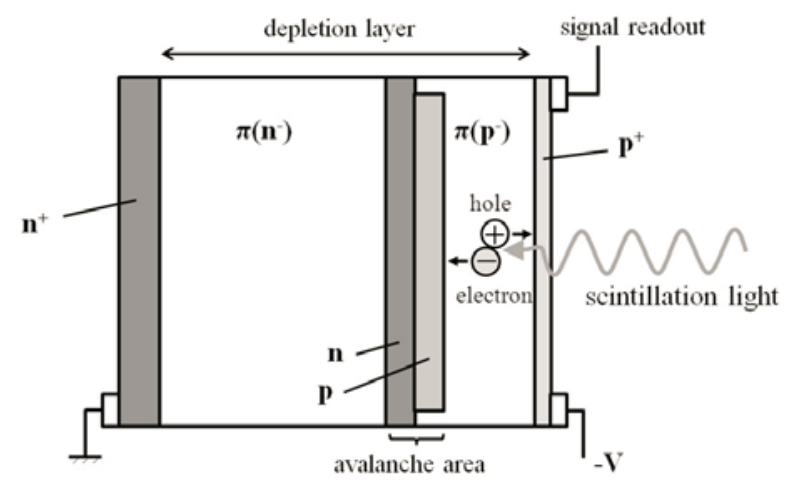

Fig. 1 The schematic structure of avalanche photodiode (APD).

formed with the bias voltage. In the avalanche area, the electrons are accelerated by the very strong electric field. The accelerated electrons cause the ionization by collision with bounded electrons. The ionization results in the internal amplification (avalanche effect).

\section{Experiments}

\section{Detection of neutrons and $\gamma$-rays}

Experimental apparatus is schematicaly shown in Fig. 2. The Li-glass scintillator, the APD, and the ${ }^{137} \mathrm{Cs} \gamma$-ray source were placed in the vacuum chamber. In order to hold the temperature of Li-glass scintillator to $77 \mathrm{~K}$, the vessel was filled with liquid nitrogen $\left(\mathrm{LN}_{2}\right)$. The ${ }^{252} \mathrm{Cf}$ neutron source was placed outside of the vessel. A block of paraffin wax of 45-mm-thick was placed between neutron source and $\mathrm{LN}_{2}$ vessel. The neutrons emitted by the ${ }^{252} \mathrm{Cf}$ source were moderated in the block of paraffin wax to increase the event of the ${ }^{6} \mathrm{Li}(n, \alpha) \mathrm{T}$ reaction.

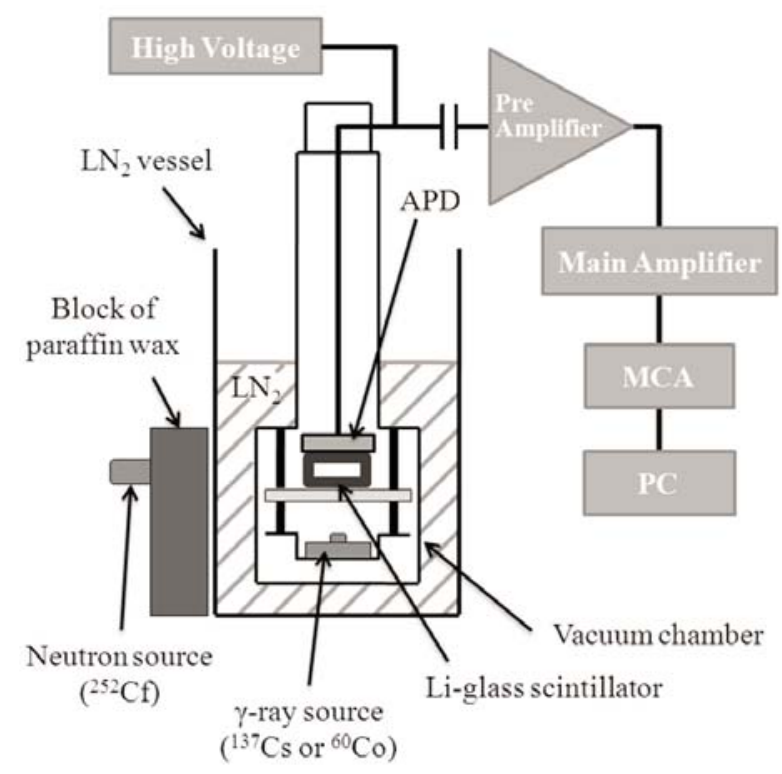

Fig. 2 The schematic structure of experimental apparatus.
The scintillation light was generated by the Li-glass scintillator in detection of neutrons and $\gamma$-rays emitted from ${ }^{252} \mathrm{Cf}$ and ${ }^{137} \mathrm{Cs}$. The scintillation light was converted into electronic charge signal by the APD. The charge signal was converted into the voltage pulse signal by the preamplifier (CAMBERRA 2003BT). Output voltage signal pulses of the preamplifier were shaped into semi-gaussian pulses and amplified by the main amplifier (ORTEC 672). The pulse height distribution was obtained by measuring the pulse height of the semi-gaussian pulses by a multi-channel pulse height analyzer (MCA: AMPTEK MCA8000). The measurements were performed at temperatures of $300 \mathrm{~K}$ and $77 \mathrm{~K}$, respectively.

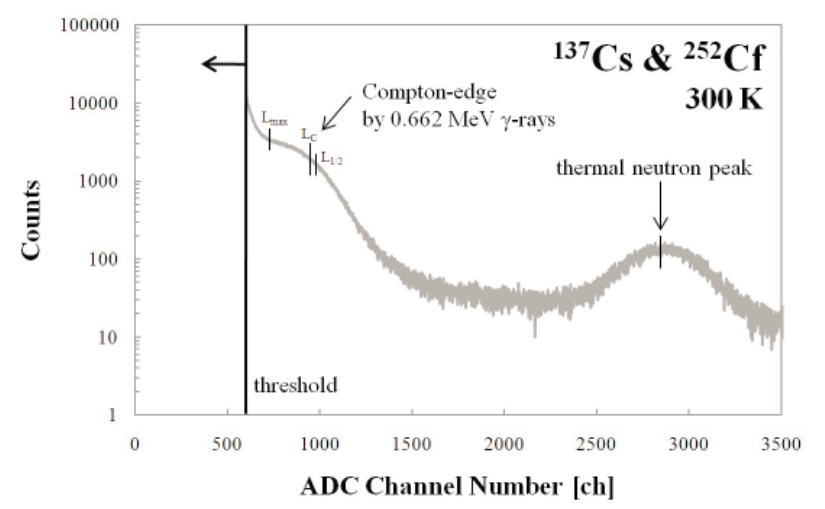

Fig. 3 Pulse height distribution of signals in detection neutrons and $\gamma$-rays emitted from ${ }^{252} \mathrm{Cf}$ neutron source and ${ }^{137} \mathrm{Cs}$ $\gamma$-ray source at $300 \mathrm{~K}$.

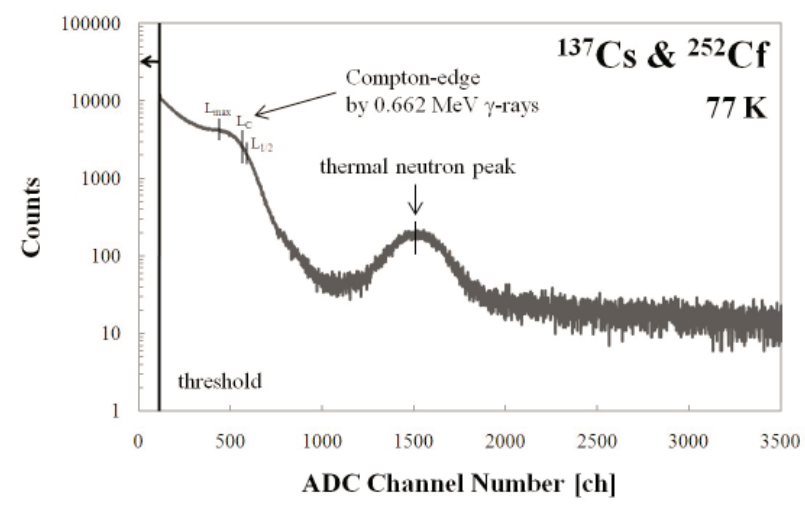

Fig. 4 Pulse height distribution of signals in detection neutrons and $\gamma$-rays emitted from ${ }^{252} \mathrm{Cf}$ neutron source and ${ }^{137} \mathrm{Cs}$ $\gamma$-ray source at $77 \mathrm{~K}$.

Figure 3 shows obtained pulse height distribution of signal in the detection of neutrons and $\gamma$-rays at $300 \mathrm{~K}$. In the measurement at $300 \mathrm{~K}$, the APD was operated with applying the bias voltage of $416 \mathrm{~V}$. Gain and shaping time of the main amplifier were selected to be 500 and $0.5 \mu \mathrm{s}$, respectively. Compton-edge by $\gamma$-rays appears in the low channel. In this experiment, the position of the Compton-edge was determined by using the empirical method for a NE213 liquid scintillator. ${ }^{4)}$ In Fig. $3 L_{\max }$ indicates a peak position in the Compton-continuum. A position of $L_{1 / 2}$ corresponds to the 
half height of $L_{\max }$. A position of the ideal Compton-edge $L_{C}$ is empirically obtained by

$$
L_{1 / 2}=1.03 L_{C}
$$

By using eq. (1), the position of Compton-edge by $\gamma$-rays emitted by ${ }^{137} \mathrm{Cs}$ was estimated to be $L_{C}=952 \pm 5 \mathrm{ch}$. In this work, the statistical error is considered in estimations. The thermal neutron peak by the ${ }^{6} \operatorname{Li}(n, \alpha) \mathrm{T}$ reaction with incidence neutrons emitted from ${ }^{252} \mathrm{Cf}$ appears at $2853 \pm 88 \mathrm{ch}$.

Figure 4 shows obtained pulse height distribution of signal in detection of neutrons and $\gamma$-rays at $77 \mathrm{~K}$. In the measurement at $77 \mathrm{~K}$, APD was operated with applying the bias voltage of $290 \mathrm{~V}$. Gain and shaping time of the main amplifier were selected to be 180 and $0.5 \mu \mathrm{s}$, respectively. The value of $L_{C}$ in irradiation with $\gamma$-rays emitted by ${ }^{137} \mathrm{Cs}$ was estimated to be $570 \pm 2 \mathrm{ch}$, and thermal neutron peak by the ${ }^{6} \mathrm{Li}(n, \alpha) \mathrm{T}$ reaction by moderated neutrons emitted by ${ }^{252} \mathrm{Cf}$ appears at $1509 \pm 56 \mathrm{ch}$. The threshold of MCA decreased with temperatures since the signal-to-noise ratio of APD grows in $77 \mathrm{~K}$ compared with $300 \mathrm{~K}$.

\section{Detection of $\gamma$-rays emitted from ${ }^{60} \mathrm{Co}$ source}

The Li-glass was irradiated with $\gamma$-rays emitted by ${ }^{60} \mathrm{Co}$ source, in order to calibrate pulse height distributions concerned with the gamma equivalent energy. In Fig. 2, ${ }^{137} \mathrm{Cs}$ $\gamma$-ray source was replaced with ${ }^{60} \mathrm{Co} \gamma$-ray source, and ${ }^{252} \mathrm{Cf}$ neutron source was removed.

Figure 5 shows obtained pulse height distribution of detection signals of $\gamma$-rays at $300 \mathrm{~K}$. In the measurement at $300 \mathrm{~K}$, the APD was operated with applying the bias voltage of $416 \mathrm{~V}$. Gain and shaping time of the main amplifier were selected to be 500 and $0.5 \mu \mathrm{s}$, respectively. The value of $L_{C}$ in irradiation with $\gamma$-rays emitted by ${ }^{60} \mathrm{Co}$ was estimated to be $1853 \pm 22 \mathrm{ch}$.

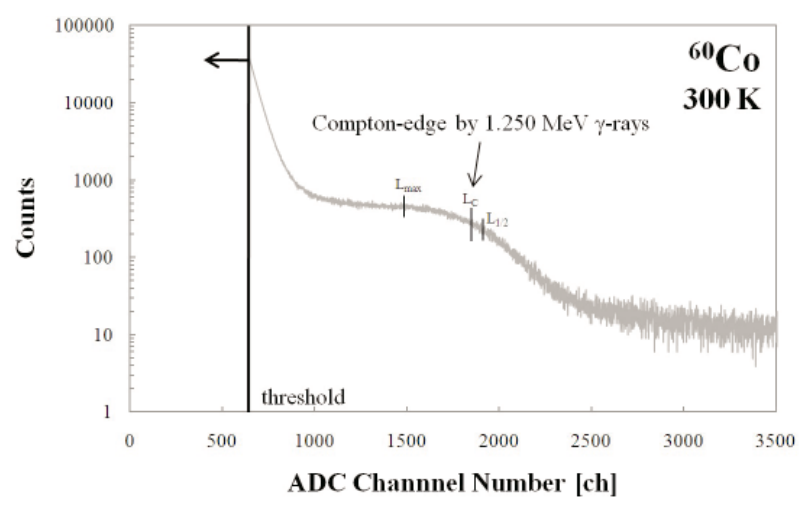

Fig. 5 Pulse height distribution of signals in detection $\gamma$-rays emitted from ${ }^{60} \mathrm{Co} \gamma$-ray source at $300 \mathrm{~K}$.

Figure 6 shows obtained pulse height distribution of detection signals of $\gamma$-rays at $77 \mathrm{~K}$. In the measurement at $77 \mathrm{~K}$, the APD was operated with applying the bias voltage of $290 \mathrm{~V}$. Gain and shaping time of the main amplifier were selected to be 180 and $0.5 \mu \mathrm{s}$, respectively. The value of $L_{C}$ in irradiation with $\gamma$-rays emitted by ${ }^{60} \mathrm{Co}$ was estimated to be $1345 \pm 15 \mathrm{ch}$. The threshold of MCA has become small.

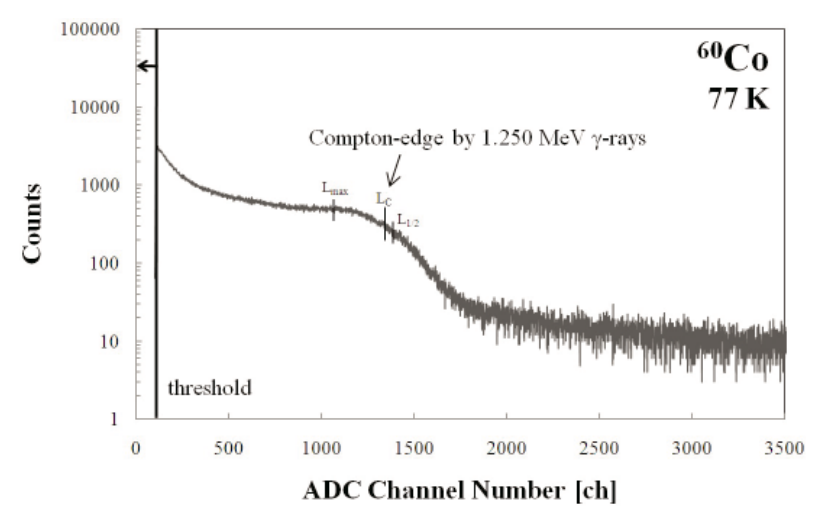

Fig. 6 Pulse height distribution of signals in detection $\gamma$-rays emitted from ${ }^{60} \mathrm{Co} \gamma$-ray source at $77 \mathrm{~K}$.

\section{Gamma equivalent energy}

The ADC channel number is converted into gamma equivalent energy in order to compare the measurement result at the temperature of $300 \mathrm{~K}$ and $77 \mathrm{~K}$. Figure 7 shows the relationship between the ADC channel number and the gamma equivalent energy. Straight lines in Fig. 7 is drawed by using the relationship between the Compton-edge energy and $L_{C}$ values obtained in Figs 3 - 6.

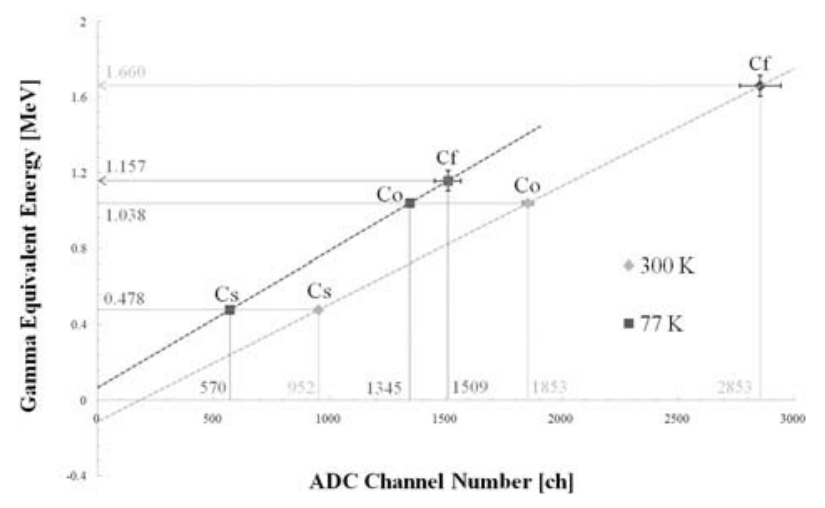

Fig. 7 Gamma equivalent energy calibration graph.

Table 2 is the list of the numerical values used for a calculation of the gamma equivalent energy calibration. The values of the gamma equivalent energy of the thermal neutron peak at temperature of $300 \mathrm{~K}$ and $77 \mathrm{~K}$ are obtained to be respectively $1.66 \pm 0.09 \mathrm{MeV}$ and $1.16 \pm 0.06 \mathrm{MeV}$ by using calibration

Table 2 Energy and position of Compton-edge.

\begin{tabular}{c|c|c}
\hline & ${ }^{137} \mathrm{Cs}$ & ${ }^{60} \mathrm{Co}$ \\
\hline \hline Energy of $\gamma$-ray & $0.662 \mathrm{MeV}$ & $1.250 \mathrm{MeV}$ \\
Energy of Compton-edge & $0.478 \mathrm{MeV}$ & $1.038 \mathrm{MeV}$ \\
Position of & $952 \pm 5 \mathrm{ch}$ & $1853 \pm 22 \mathrm{ch}$ \\
$\begin{array}{c}\text { Compton-edge at } 300 \mathrm{~K} \\
\text { Position of }\end{array}$ & $570 \pm 2 \mathrm{ch}$ & $1345 \pm 15 \mathrm{ch}$ \\
Compton-edge at $77 \mathrm{~K}$ & & \\
\hline
\end{tabular}


lines in Fig. 7. The values corresponding to the thermal neutron peak are also plotted in Fig. 7.

Figure 8 shows pulse height distributions concerned with gamma equivalent energy of signals in detection neutrons and $\gamma$-rays emitted from ${ }^{252} \mathrm{Cf}$ neutron source and ${ }^{137} \mathrm{Cs} \gamma$-ray source at temperature of $300 \mathrm{~K}$ and $77 \mathrm{~K}$. The gamma equivalent energy of the thermal neutron peak at $77 \mathrm{~K}$ decreases to $70 \pm 7 \%$ of those at $300 \mathrm{~K}$. The ratios of the light yield in thermal neutron detection to the light yield in $\gamma$-ray detection at temperature of $300 \mathrm{~K}$ and $77 \mathrm{~K}$ are obtained to be $35 \pm 2 \%$ and $24 \pm 1 \%$, respectively. Detection performance of Li-glass scintillator was found not to be improved with decreasing temperature.

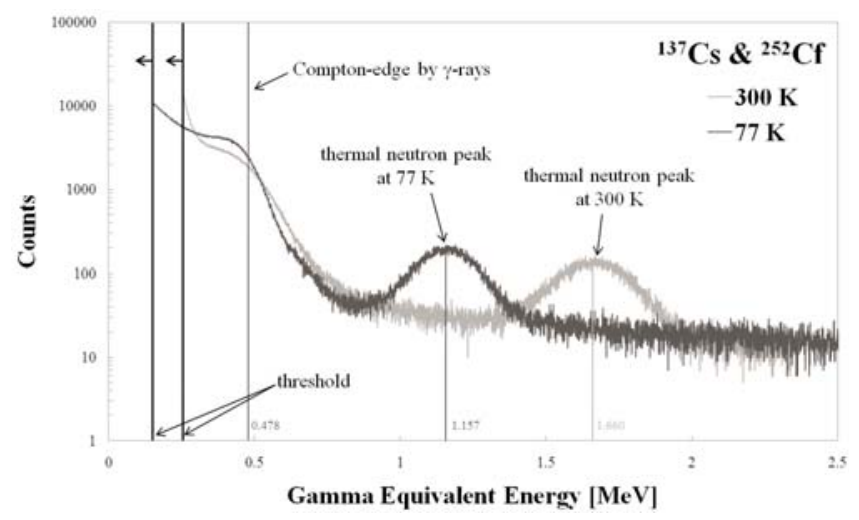

Fig. 8 Pulse height distributions concerned with gamma equivalent energy of signals in detection neutrons and $\gamma$-rays emitted from ${ }^{252} \mathrm{Cf}$ neutron source and ${ }^{137} \mathrm{Cs} \gamma$-ray source at temperature of $300 \mathrm{~K}$ and $77 \mathrm{~K}$.

\section{Conclusion}

The pulse height distributions of detection signals of Liglass scintillator were obtained by irradiation with neutrons and $\gamma$-rays at temperatures of $300 \mathrm{~K}$ and $77 \mathrm{~K}$. For the Liglass scintillator, the ratio of the light yield in thermal neutron detection to that in $\gamma$-ray detection was found to decrease with temperatures. The decrease in the relative light yield of neutron detection would be caused by a reduction in numbers of the excited electron which contributes to the scintillation luminescence.

\section{References}

1) R.B. Murray, "Use of $\mathrm{Li}^{6} \mathrm{I}(\mathrm{Eu})$ as a scintillation detector and spectrometer for fast neutrons," Nuclear Instruments, 2[3], 237 (1958).

2) L. Yang, S. N. Dzhosyuk, J. M. Gabrielse, et al., "Performance of a large-area avalanche photodiode at low temperature for scintillation detection," Nuclear Instruments and Methods in Physics Research, A508[3], 388 (2003).

3) A. Dorokhov, A. Glauser, Y. Musienko, et al., "Study of the Hamamatsu avalanche photodiode at liquid nitrogen temperatures," Nuclear Instruments and Methods in Physics Research, A504[1-3], 58 (2003).

4) G. Dietze and H. Klein, "Gamma-calibration of NE 213 scintillation counters," Nuclear Instruments and Methods in Physics Research, 193, 549 (1982). 